\title{
The problem of collective identity in a fuzzy environment
}

\author{
José Carlos R. Alcantud ${ }^{1, *}$ and Rocío de Andrés Calle ${ }^{2}$ \\ 1 BORDA Research Unit and Multidisciplinary Institute of Enterprise (IME) \\ University of Salamanca, Spain \\ jcr@usal.es \\ ${ }^{*}$ Corresponding author \\ 2 BORDA Research Unit, PRESAD Research Gorup and \\ Multidisciplinary Institute of Enterprise (IME) \\ University of Salamanca, Spain \\ rocioac@usal.es
}

\begin{abstract}
We define the problem of group identification in a fuzzy environment. We concentrate on the case where the society is required to self-determine the belongingness of each member to a specific group, characterized by a single attribute. In general terms, this case consists of a collective identity issue that can be regarded as an aggregation problem of individual assessments within a group. Here we introduce the possibility that both the original assessments and the resulting output attach partial memberships to the collectivity, for each potential member. We propose relevant classes of rules, and some are axiomatically characterized. Our new approach provides a way to circumvent classical impossibility results like Kasher and Rubinstein's.
\end{abstract}

Keywords: Group identification; Fuzzy profile; Fuzzy collective identity function; Choquet Fuzzy collective identity function; WOWA-Fuzzy collective identity function.

\section{Introduction}

The purpose of this paper is to extend the study of the group identification problem in Social Choice by allowing the natural possibility of partial membership degrees of the individuals to the groups. To be more precise in this first work we focus on a clear-cut statement called the problem of collective identity.

In this regard we adhere to Kasher [23], who introduces a notion of collective identity that remains basically unchallenged. It first attempts to answer the question of how to define a social group, albeit in a non-formalized sense. Kasher and Rubinstein [24] build on this philosophical position and state the problem from the point of view of Social Choice. To this purpose, they pose the question as an aggregation problem where the views of every member of the society about every member determine a well-defined collective identity of the group. In formal 
terms, the social outcome of their problem is a (crisp) subset of the society. The success of their contribution is that they show how algebraic aggregation theory (cf., Rubinstein and Fishburn [31]) intervenes to provide answers to such problem about aggregation of individual opinions. Among other achievements, they prove a celebrated impossibility theorem under fairly mild conditions. Therefore although the use of aggregators (in the sense of [23] or [24]) and of aggregation operators (e.g., in the algebraic framework posed by [31] in economic theory) is not the same, the first relates to the second at the outset.

Clearly, an inspection of the relevant literature shows that the modeling of how individuals in a society are collectively viewed as belonging to a group is naturally linked to vague attributes, e.g., 'belonging to a newly formed nationality' (Dimitrov, Sung and Xu [16]), 'being Jewish' (Kasher [23]), 'being African-American' or belonging to any racial group (Miller, [28]), 'living in a rich neighborhood' (Dimitrov [14]), etc. Nevertheless the Social Choice literature has almost universally neglected this fact in the analysis of how social groups should be defined. By incorporating the subjectivity associated with general and vague attributes in the form of partial memberships we provide a richer environment where either the personal views of the individuals or the resulting collective identity or both are not necessarily dichotomic any more. In this framework we can better capture the subtleties of the problem, also because new families of aggregation operators can be employed to determine the social outcome (which can now be a fuzzy subset of the society).

Regarding this issue, besides the few traditional aggregation rules from the Social Choice literature we introduce the discrete Choquet integral, ordered weighted averaging operators (OWA), and weighted OWA operators (WOWA) as new tools that may determine the degree of memberships of the individuals to the collective identity. We utilize these aggregation functions due to their versatility and their well-known properties, that we proceed to briefly review. ${ }^{3}$

The Choquet integral is the integral of a real function with respect to a fuzzy measure, by analogy with the Lebesgue integral which is defined with respect to an ordinary (i.e., additive) measure. Under the premise that the measure of the whole set is one, the discrete Choquet integral can be regarded as a mean or an averaging aggregation operator. In fact Marichal [26] presents the Choquet integral as an appropriate extension to the weighted arithmetic mean for the aggregation of criteria. Although it offers a large flexibility, somehow it keeps a linear form. Marichal proves how the Choquet integral can be characterized axiomatically by means of natural properties in [26, Section IV.B]. He offers an intuitive approach to it in [26, Section IV.A]. Several graphical interpretations of the Choquet integral are available from the literature, e.g., in Grabisch et al. [21] and Torra and Narukawa [37]. Earlier uses of Choquet integrals as aggregation functions in other contexts can be found e.g., in Calvo, Mayor and Mesiar [9,

\footnotetext{
${ }^{3}$ Other developments on collective aggregation of fuzzy sets or fuzzy relations from Decision Making literature can be found in Barret [5], Barret, Pattanaik and Salles [6], Dubois and Koning [17] and Montero [29], among others,
} 
pp. 224-244], Grabisch [18], Grabisch et al. [19], Grabisch and Labreuche [20], and Marichal [27].

A particular instance of the discrete Choquet integral is the OWA operator (cf., Yager [38]). It is similar to a weighted mean, which synthesizes the values of the information sources according to their respective reliabilities, the difference being that in OWAs the values of the variables are previously ordered in a decreasing way. Therefore now the weights are not associated with concrete variables, and OWAs can diminish the importance of extreme values in order to increase the relevance of central ones. Calvo, Mayor and Mesiar [9], de Andrés Calle et al. [13], Llamazares [25] (in the context of Social Choice), and Yager and Kacprzyk [40] among others supply examples of applications of the OWA operator. From the point of view of the aggregation of opinions, it preserves anonymity while keeping the attractive of different weights.

An aggregation procedure that combines the advantages of using weights both to discriminate the sources of information and to calibrate the relevance of values in relation to their relative position is the WOWA operator (cf., Torra [34]). This procedure relies on an interpolation method (see also Torra [35]).

We can now be more explicit about the main target of this paper. We provide evidences that extending the analysis of the problem of collective identity by the introduction of partial memberships permits to better capture the nuances of preference modeling. Several frameworks apropos the fuzzy environment are presented and their properties are studied. We show that it is possible to preserve the nature of the investigations in Social Choice (e.g., the axiomatic treatment of the models) and to avoid restrictions imposed by the dichotomic viewpoint of the problem. We prove these benefits by characterizing the rules that evaluate each individual's membership to the collectivity by a discrete Choquet integral, and by producing a positive escape from Kasher and Rubinstein's impossibility theorem.

The outline of our exposition is as follows. Section 2 explains the origin of the problem, its antecedents, and the precise framework in terms of crisp or categorical opinions that we investigate. In Section 3 we define our model for the fuzzy collective identity problem, allowing for various degrees of generalization. Our main tool of analysis, namely, fuzzy collective identity functions (FCIFs), is put forward and some direct examples are presented which extend the traditional collective identity functions. Then in Section 4 we propose a newly designed approach which determines belongingness to the collectivity by Choquet-FCIFs or WOWA-FCIFs. Both include interesting particular cases like $O W A$-FCIFs, the average mean FCIF, or weighted mean FCIFs. We import properties of the corresponding aggregation operators in order to derive properties of these FCIFs. Some examples illustrate their use too. In Section 5 we set out some general conclusions as well as a possible circumvention of Kasher and Rubinstein's impossibility theorem. In addition we provide a characterization of Choquet-FCIFs that derives from a known axiomatization of the discrete Choquet integral. Moreover, we present several ideas for future research. 


\section{Antecedent and current state of the problem}

The problem of the identification and qualification of individuals is one of the main issues in social and economic contexts. In this section we give an overview of how the problem has been addressed in this literature and then formalize the framework that motivates our study.

\subsection{Related literature}

Kasher [23] first analyzes the collective identity question from a philosophical perspective but recognizes it as an aggregation problem of individual assessments. Every agent in a society gives their opinion on herself/himself and other agents regarding a particular attribute ${ }^{4}$. Then an aggregation function determines the agents belonging to the collective in a recursive procedure, taking into account their individual views ${ }^{5}$.

Subsequently, Kasher and Rubinstein [24] establish a link between the concept of collective identity proposed by Kasher and the algebraic theory of aggregators. These authors provide axiomatic characterizations for three extreme aggregation rules: the strong liberal, the dictatorships and the oligarchical rule. Saporiti [33] gives a direct proof of the characterization of dictatorial rules in the tradition of earlier proofs of Arrow's impossibility theorem [2].

Samet and Schmeidler [32] study and characterize a class of voting rules, called consent rules. These rules incorporate aspects of majoritarianism and liberalism. They follow [24] to study the relation between the liberal and the majoritarian rules in the same mode.

In relation with the problem of endogeneos classification, already Kasher and Rubinstein [24, Section 6] consider the environment where every person has a position about how the society should be partitioned into non-ranked classes. In this respect the group identification problem addresses the issue of constructing a decomposition of the group into classes from such profiles. Some scholars have studied the case in which the number of classes is fixed (but not necessarily reduced to two as in the collective identity problem), e.g., Houy [22, Section 2] and Miller [28]. Others have been concerned with the case in which the individuals only express their wishes about who should be put together in the same classes, e.g., the aforementioned Kasher and Rubinstein [24, Section 6], Houy [22, Section 3] and Dimitrov and Puppe [15].

Some other contributions include Ballester and García-Lapresta [3], Billot [7], Çengelci and Sanver [10], or Dimitrov, Sung and Xu [16]. Table 1 summarizes these and other works. Dimitrov [14] constitutes a nice survey of the various approaches to the group identification problem.

Now we discuss the common framework in the aforementioned contributions.

\footnotetext{
${ }^{4}$ Kasher focuses on Jewish identity.

${ }^{5}$ Kasher only considers aggregation methods that satisfy Rawls' justice principle [30].
} 
Collective Identity (one attribute characterizes identity)

\begin{tabular}{ll}
\hline Author(s)/Year & Decision Rules \\
\hline Kasher [23], 1993 & Philosophical point of view \\
Kasher and Rubinstein [24], 1997 & Liberal and Dictatorial \\
Samet and Schmeidler [32], 2003 & Consent rules, Liberal (2 axiomatizations) \\
Billot [7], 2003 & Qualification \\
Dimitrov et. al [16], 2007 & Consensus-start-respecting \\
& and Liberal-start-respecting \\
Ballester and García-Lapresta [3], 2008 & Elitist qualification \\
Çengelci and Sanver [10], 2010 & Simple, Liberal (2 axiomatizations) \\
Saporiti [33], 2012 & Dictatorial \\
&
\end{tabular}

Group Identification (society self-determines how its members are classified into groups)

\begin{tabular}{ll}
\hline Author(s)/Year & Decision Rules \\
\hline Kasher and Rubinstein [24], 1997 & Oligarchy \\
Houy [22], 2007 & Liberal (name of group matters) \\
& Universal, Joint and Almost joint \\
Miller [28], 2008 & Agreement, Nomination and One-vote rules \\
Dimitrov and Puppe [15], 2011 & Non-bossy \\
\hline
\end{tabular}

Table 1: Summary table of studies related to endogenous selection.

\subsection{Standing framework for endogenous selection: the case of a single group}

Virtually all the cited references have approached endogeneous selection from a dichotomous perspective. Individuals only show extreme or 'crisp' opinions on the possible belongingness of each individual to the group. The resulting social outcome (i.e., the final self-selected collectivity) suffers from the same limitation. Thus in the case of self-selecting the members of one group (e.g., the persons that verify a given attribute like being African-American), both in the opinions expressed by each member of the society and in the final outcome it is assumed that each individual either belongs or does not belong to the referred group. ${ }^{6}$ From a formal viewpoint, the case where one attribute characterizes identity claims that the $N=\{1, \ldots, n\}$ agents face the problem of collectively choosing a subset of $N$. And that the input for this collective selection is their individual opinions concerning who qualifies as a member of such self-selected group. These opinions are summarized by (crisp) profiles. A profile is an $n \times n$ matrix $P=\left(P_{i j}\right)_{n \times n}$, the cells of which are either 0 or 1 throughout. The set of all

\footnotetext{
${ }^{6}$ As an exception, Ballester and García-Lapresta [4] define a restrictive concept called "committee's evaluation mechanism" that determines individual qualification only in gradual terms. However this is an intermediate step prior to the construction of a categorical "committee's qualification mechanism" via a fixed family of thresholds. The latter mechanism generates a subset of qualified individuals in each stage of a recursive procedure that may not converge (cf., [4, Corollary 1]).
} 
such matrices is denoted by $\mathcal{M}$. We say that agent $i$ qualifies agent $j$ if and only if $P_{i j}=1$. And this means that in person $i$ 's opinion, person $j$ verifies the attribute that defines the collective identity when $P_{i j}=1$, and does not verify it when $P_{i j}=0$.

For each set $S$, let $\mathcal{P}(S)$ denote the set of subsets of $S$. Row $i$ in $P \in \mathcal{M}$ captures the group of qualified agents in $i$ 's opinion. It can be easily identified with a subset $J_{i}(P) \in \mathcal{P}(N)$ through the expression: $j \in J_{i}(P)$ if and only if $P_{i j}=1$. And column $j$ describes who are the agents who qualify $j$.

Definition 1 (Kasher and Rubinstein [24]). A collective identity function $(C I F)$ is a mapping $\mathcal{J}: \mathcal{M} \longrightarrow \mathcal{P}(N)$. Thus $\mathcal{J}$ associates with each profile $P \in \mathcal{M}$ a subset of $N$, which can be naturally identified with an $n$-vector of 0 's and 1 's as done above.

CIFs attach a (crisp) subset of the society $N$ with each collection of individual opinions on who should belong to the self-selected group. It is only natural to introduce partial memberships (to the group that verifies the required attribute) in the model by Kasher and Rubinstein. Hence in Section 3 below we extend this framework to the fuzzy environment. There we formulate some naive identification rules that in particular extend the fundamental solutions to the problem of group identification as stated above. Subsequently, Section 4 below develops new sophisticated analyses and methodologies.

\section{The problem: group identification in fuzzy environments}

We proceed to model the problem of endogenous selection from a new perspective that includes the original statement of the issue. Our aim is twofold. We introduce the problem of group identification when both the input (individual opinions) and the outcome (aggregation opinions) are fuzzy. This is the most general case due to the fact that the agents can express their opinions on the degree of belongingness to the group of each agent, and then degrees of partial membership are obtained as a result. Then, we present some fundamental definitions of focal criteria that are motivated by the existing literature.

In addition we consider two partial frameworks where inputs or outputs are crisp or fuzzy. Concretely, we introduce the issue when the input is crisp (in the standard sense of Section 2) but the result is fuzzy. That is to say, although the agents must be categorical on exactly who qualifies as a member of the group, the resulting membership function of the agents is fuzzy (degrees of partial membership are allowed). We also make some remarks on the particular case where the opinions of the agents are fuzzy but the verdict is crisp.

\subsection{Fuzzy CIFs (FCIFs)}

Henceforth $\operatorname{FS}(S)$ denotes the fuzzy subsets (FSs) of the set $S$. We can embed $\mathcal{P}(S)$ into $\mathrm{FS}(S)$ through the standard identification. 
When the $N=\{1, \ldots, n\}$ agents face the problem of collectively determining a fuzzy subset of $N$, one should also investigate the situation where the agents are allowed to attach partial memberships to each individual. These opinions are summarized by fuzzy profiles.

A fuzzy profile is an $n \times n$ matrix $M=\left(M_{i j}\right)_{n \times n}$, the cells of which are numbers in the interval $[0,1]$.

$$
M=\left(\begin{array}{ccc}
M_{11} & \ldots & M_{1 n} \\
\vdots & \ddots & \vdots \\
M_{n 1} & \ldots & M_{n n}
\end{array}\right)_{n \times n}
$$

We interpret that agent $i$ qualifies agent $j$ as a member of the collectivity with degree of belongingness $M_{i j}$. Now this means that in person $i$ 's opinion, person $j$ verifies the attribute that defines the collective identity with a degree of $M_{i j}$. Consequently, column $j$ of the profile $M$ captures the opinions on the degree of belongingness to the group of indivual $j$. We denote it by $M^{(j)}$. We write $\mathbf{M}$ for the set of all $n \times n$ matrices.

Any permutation $\pi$ of the agents $\{1,2, \ldots, k\}$ determines a fuzzy profile ${ }^{\pi} M$ by permutation of the columns of $M$ : column $j$ of the profile ${ }^{\pi} M$ is column $\pi(j)$ of the profile $M$.

By incorporating the expression of partial memberships we allow the agents to provide more precise information than in the standard model (cf., subsection 2.2 ). The recourse to fuzzy profiles permits to extend Definition 1 in the following novel way:

Definition 2. A fuzzy CIF (FCIF) is a mapping $\boldsymbol{J}: \mathbf{M} \longrightarrow \mathrm{FS}(N)$, which associates with each fuzzy profile $M \in \mathbf{M}$ a fuzzy subset of $N$.

FCIFs express to which degree the agents belong to the self-selected group as a function of the fuzzy opinions by all agents on that issue.

We proceed to propose the following new definitions of potentially useful FCIFs that are inspired by respective notions from the Social Choice literature:

Definition 3. The liberal FCIF is defined as $\boldsymbol{J}_{l}: \mathbf{M} \longrightarrow \mathrm{FS}(N)$ such that for each $M \in \mathbf{M}$,

$$
\boldsymbol{J}_{l}(M)(i)=M_{i i} \in[0,1], \text { for each } i \in N .
$$

Definition 4. The dictatorial FCIF by agent $j$ is defined as as follows: $\boldsymbol{J}_{d_{j}}: \mathbf{M} \longrightarrow \mathrm{FS}(N)$ such that for each $M \in \mathbf{M}$,

$$
\boldsymbol{J}_{d_{j}}(M)(i)=M_{j i}, \text { for each } i \in N \text {. }
$$

Definition 5. The oligarchical FCIF by the set of agents $O \subseteq N$ is defined as $\boldsymbol{J}_{o}: \mathbf{M} \longrightarrow \mathrm{FS}(N)$ such that for each $M \in \mathbf{M}$,

$$
\boldsymbol{J}_{o}(M)(i)=\min _{j \in O} M_{j i}, \text { for each } i \in N \text {. }
$$


Definitions 3, 4 and 5 respectively extend the liberal, dictatorial and oligarchical CIFs originally defined by Kasher and Rubinstein $[24]^{7}$. According to the liberal FCIF, every person decides on her own degree of belongingness to the self-selected collectivity. The dictatorial FCIF is used when one person (the dictator) decides on everyone's degree of belongingness to the group. Relatedly, under the oligarchical FCIF there is a fixed part of the society that decides on everyone's degree of belongingness to the group by agreement on her minimum degree of membership. Therefore the oligarchical FCIF is a dictatorial FCIF when the oligarchy $O$ consists of a single agent (the dictator). And the case when the oligarchy $O$ is the whole society produces the following remarkable case:

Definition 6. The conjunctive FCIF is defined as $\boldsymbol{J}_{c}: \mathbf{M} \longrightarrow \mathrm{FS}(N)$ such that for each $M \in \mathbf{M}$,

$$
\boldsymbol{J}_{c}(M)(i)=\min _{j \in N} M_{j i}, \text { for each } i \in N .
$$

It is just natural to define the counterpart of $\mathbf{J}_{c}$ as follows:

Definition 7. The benevolent FCIF is defined as $\boldsymbol{J}_{b}: \mathbf{M} \longrightarrow \mathrm{FS}(N)$ such that for each $M \in \mathbf{M}$,

$$
\boldsymbol{J}_{b}(M)(i)=\max _{j \in N} M_{j i}, \text { for each } i \in N .
$$

The conjunctive FCIF declares that an individual belongs to the collectivity with the larger degree of membership on which everyone in the society agrees (in the sense: everyone believes that his or her degree of membership is at least as large as such indicator of belongingness). Thus it implements a very restrictive view of group identity. Relatedly, the benevolent FCIF declares that an individual belongs to the collectivity with the larger degree of membership declared by any of the members thus it captures a very permissive view of group identity.

The conjunctive FCIF extends the spirit of the joint or conjunctive CIF that Houy [22, Definition 3] characterizes in the context of group identification with partitions as inputs. In fact it is the most studied rule in the context of aggregating partitions (cf., Dimitrov and Puppe [15, Section 1]). Houy's statement declares that two individuals are in the same group if and only if each individual wants them to be in the same group.

We recall that the empty FS of $N$ is $\mathbf{0}: N \longrightarrow[0,1]$ such that $\mathbf{0}(i)=0$ for each $i \in N$. The full FS of $N$ is $\mathbf{1}: N \longrightarrow[0,1]$ such that $\mathbf{1}(i)=1$ for each $i \in N$. Then we can define two extreme or degenerate instances:

Definition 8. The empty FCIF is defined as $\boldsymbol{O}: \mathbf{M} \longrightarrow \mathrm{FS}(N)$ such that $\boldsymbol{O}(M)=\boldsymbol{O}$ for each $M \in \mathbf{M}$. The universal FCIF is defined as $\mathbf{1}: \mathbf{M} \longrightarrow \mathrm{FS}(N)$ such that $\mathbf{1}(M)=\mathbf{1}$ for each $M \in \mathbf{M}$.

\footnotetext{
${ }^{7}$ However in the case of the oligarchical aggregator, they rather think of a task partitioning all members of the society into an unlimited number of classes. This is a different model: see e.g., Houy [22] for differences between the two approaches.
} 
The empty (resp., universal) FCIF declares that irrespective of the opinions of the agents, the degree of membership of each agent to the selected group is null (resp., full). The universal FCIF extends the spirit of the universal CIF (e.g., Houy [22, Definition 2]).

Now we proceed to define some new particular specifications of FCIFs that take full advantage of the fuzzy structure of the problem.

Definition 9. Let us fix $\boldsymbol{w}=\left(w_{1}, \ldots, w_{n}\right) \in[0,1]^{n}$ weighting vector with $\sum_{i=1}^{n} w_{i}=1$. The weighted mean FCIF associated with $\boldsymbol{w}$ is defined as the mapping $\boldsymbol{J}^{\boldsymbol{w}}: \mathbf{M} \longrightarrow \mathrm{FS}(N)$ such that for each $M \in \mathbf{M}$,

$$
\boldsymbol{J}^{\boldsymbol{w}}(M)(i)=\sum_{j \in N}\left(w_{j} M_{j i}\right), \text { for each } i \in N \text {. }
$$

In particular, the average (or arithmetic) mean FCIF is defined as follows:

$$
\boldsymbol{J}_{a}(M)(i)=\frac{\sum_{j \in N} M_{j i}}{n} \text { for each } i \in N \text { and } M \in \mathbf{M} \text {. }
$$

The average FCIF assesses the degree of membership of a person by the average of the opinions on her belonging to the selected group by all the agents. This means that the opinions of all agents are interchangeable. When we value the opinions of the agents differently (e.g., due to their expertise or position or share in a company) then we can use an adequate weighted mean FCIF instead.

Definition 10. The majoritarian FCIF is defined as $\boldsymbol{J}_{m}: \mathbf{M} \longrightarrow \mathrm{FS}(N)$ such that for each $M \in \mathbf{M}$,

$$
\boldsymbol{J}_{m}(M)(i)= \begin{cases}1 & \text { if } \sum_{j \in N} M_{j i}>\frac{n}{2}, \\ \frac{\sum_{j \in N} M_{j i}}{n} & \text { otherwise. }\end{cases}
$$

The majoritarian FCIF imports the ethos of the majoritarian rule studied by Samet and Schmeidler [32] and Çengelci and Sanver [10]. It declares that when the aggregate membership degree for one person is larger than one half of the maximum possible aggregate, her degree of membership to the selected group is 1. Otherwise it is assessed by the arithmetic mean. Thus it can be regarded as a correction of $\mathbf{J}_{a}$ where agents with sufficiently high total membership degree are awarded a bonus of full membership.

Now we introduce a brief example in order to put in practice the proposed definitions.

Example 1. Consider the following fuzzy profile $M^{\prime} \in \mathbf{M}$ :

$$
M^{\prime}=\left(\begin{array}{rrrrrr}
0.7 & 0.9 & 0 & 0.5 & 0.6 & 0.2 \\
0.6 & 1 & 0 & 0.4 & 0.7 & 0.5 \\
0.8 & 0.8 & 0 & 0.6 & 0.5 & 0.3 \\
0.6 & 0.7 & 0.2 & 0.4 & 0.5 & 0.4 \\
0.9 & 0.9 & 0.2 & 0.3 & 0.8 & 0.3 \\
0.6 & 0.7 & 0.3 & 0.5 & 0.5 & 0.1
\end{array}\right)
$$


- The application of Definition 3 produces:

$$
\begin{aligned}
& \mathbf{J}_{l}\left(M^{\prime}\right)(1)=0.7, \mathbf{J}_{l}\left(M^{\prime}\right)(2)=1, \mathbf{J}_{l}\left(M^{\prime}\right)(3)=0 \\
& \mathbf{J}_{l}\left(M^{\prime}\right)(4)=0.4, \mathbf{J}_{l}\left(M^{\prime}\right)(5)=0.8, \mathbf{J}_{l}\left(M^{\prime}\right)(6)=0.1
\end{aligned}
$$

- The application of Definition 4 relative to agent 2 as a dictator produces:

$$
\begin{aligned}
& \mathbf{J}_{d_{2}}\left(M^{\prime}\right)(1)=0.6, \mathbf{J}_{d_{2}}\left(M^{\prime}\right)(2)=1, \mathbf{J}_{d_{2}}\left(M^{\prime}\right)(3)=0 \\
& \mathbf{J}_{d_{2}}\left(M^{\prime}\right)(4)=0.4, \mathbf{J}_{d_{2}}\left(M^{\prime}\right)(5)=0.7, \mathbf{J}_{d_{2}}\left(M^{\prime}\right)(6)=0.5 .
\end{aligned}
$$

- The application of Definition 5 relative to the oligarchy $O=\{5,6\}$ produces:

$$
\begin{aligned}
& \mathbf{J}_{o}\left(M^{\prime}\right)(1)=0.6, \mathbf{J}_{o}\left(M^{\prime}\right)(2)=0.7, \mathbf{J}_{o}\left(M^{\prime}\right)(3)=0.2 \\
& \mathbf{J}_{o}\left(M^{\prime}\right)(4)=0.3, \mathbf{J}_{o}\left(M^{\prime}\right)(5)=0.5, \mathbf{J}_{o}\left(M^{\prime}\right)(6)=0.1
\end{aligned}
$$

- The application of Definition 6 produces:

$$
\begin{aligned}
& \mathbf{J}_{c}\left(M^{\prime}\right)(1)=0.6, \mathbf{J}_{c}\left(M^{\prime}\right)(2)=0.7, \mathbf{J}_{c}\left(M^{\prime}\right)(3)=0 \\
& \mathbf{J}_{c}\left(M^{\prime}\right)(4)=0.3, \mathbf{J}_{c}\left(M^{\prime}\right)(5)=0.5, \mathbf{J}_{c}\left(M^{\prime}\right)(6)=0.1
\end{aligned}
$$

- The application of Definition 7 produces:

$$
\begin{aligned}
& \mathbf{J}_{b}\left(M^{\prime}\right)(1)=0.9, \mathbf{J}_{b}\left(M^{\prime}\right)(2)=1, \mathbf{J}_{b}\left(M^{\prime}\right)(3)=0.3 \\
& \mathbf{J}_{b}\left(M^{\prime}\right)(4)=0.6, \mathbf{J}_{b}\left(M^{\prime}\right)(5)=0.8, \mathbf{J}_{b}\left(M^{\prime}\right)(6)=0.5
\end{aligned}
$$

- The application of Definition 10 produces:

$$
\begin{aligned}
& \mathbf{J}_{m}\left(M^{\prime}\right)(1)=1, \mathbf{J}_{m}\left(M^{\prime}\right)(2)=1, \mathbf{J}_{m}\left(M^{\prime}\right)(3)=\frac{0.7}{6} \\
& \mathbf{J}_{m}\left(M^{\prime}\right)(4)=0.45, \mathbf{J}_{m}\left(M^{\prime}\right)(5)=1, \mathbf{J}_{m}\left(M^{\prime}\right)(6)=0.3 .
\end{aligned}
$$

\subsection{Weak Fuzzy CIFs (WFCIFs)}

In this subsection we restrict the framework in Definition 2 in order to avoid gradual opinions in the input of the model.

Definition 11. A weak fuzzy CIF (WFCIF) is a mapping $\tilde{\boldsymbol{J}}: \mathcal{M} \longrightarrow \operatorname{FS}(N)$, which associates with each profile $P \in \mathcal{M}$ a fuzzy subset of $N$.

WFCIFs model to which degree the agents belong to the self-selected group as a function of the categorical opinions by all agents on that issue.

Any CIF can be naturally regarded as a WFCIF. And because $\mathcal{M}$ is included into $\mathbf{M}$, the restriction of a FCIF to $\mathcal{M}$ naturally defines a WFCIF.

Similarly, Definitions 3 to 10 can be adapted to this framework by restricting their domain to $\mathcal{M}$. Intuitive interpretations are immediate. For example, now the conjunctive WFCIF declares that an individual belongs to the collectivity (with full membership) if and only if every member of the society thinks so.

Observe that the choice of partial memberships in the resulting group already permits to use rules that would be meaningless in the context of CIFs, like Definition 10. 


\subsection{Crisp qualifications from fuzzy profiles}

It is possible to specify a fourth possible framework for the endogenous selection problem, which adds to CIFs, WFCIFs, and FCIFs. In this paper we do not explicitly investigate the case where the definition of membership to the collective identity is crisp although the agents can evaluate each other with partial memberships. Of course this is a particular instance of FCIFs where the codomain is constrained. Specifically, it concerns mappings of the form

$$
J: \mathbf{M} \longrightarrow \mathcal{P}(N)
$$

which associate with each fuzzy profile $M \in \mathrm{M}$ a subset of $N .^{8}$ Therefore we do not pursue this point further (see the concluding subsection 5.3).

\section{FCIFs: Further specifications.}

The introduction of fuzzy profiles as an input of the problem of group identification permits to set forth completely new approaches to the issue. In particular, the researcher has now recourse to weighted means (which generalize arithmetic means, see Definition 9) and their extension by weighted quasi-arithmetic means (particularly, quasi-arithmetic means), to OWA (ordered weighted average) operators, and many other aggregation operators from the specialized literature like WOWA or discrete Choquet integrals (which generalize both OWA and weighted means). We proceed to develop some of these possibilities.

\subsection{The family of Choquet-FCIFs.}

Choquet integral-based aggregation seems especially appealing for the purpose of merging individual opinions to produce collective opinions. The reason is that it combines them in a way that not only considers individual inputs (as in weighted means) and their magnitude (as in OWA), but also the importance of group opinions. In our context, even if a particular opinion is deemed irrelevant by itself, still it may become very significant in the presence of other opinions. Discrete fuzzy measures allow the practitioner to attach degrees of relevance to all possible collections of opinions, thus providing flexibility for our model to a large extent.

Let us recall the elements in the definition of the discrete Choquet integral:

Definition 12. (Calvo et al. [9]) Let $N=\{1, \ldots, n\}$. A discrete fuzzy measure is a set function $\mu: 2^{N} \longrightarrow[0,1]$ which is monotonic (i.e. $\mu(S) \leqslant \mu(T)$ whenever $S \subseteq T$ ) and satisfies $\mu(\varnothing)=0, \mu(N)=1$.

\footnotetext{
${ }^{8}$ Ballester and García-Lapresta [3, Definition 1] use a related notion. They only consider mappings that derive from "collective assessment functions" (where the final set of qualified members results from a certain sequential process). Their companion paper [4] makes further use of a related, more restrictive concept as a technical tool for the construction of a crisp "committee's qualification mechanism".
} 
Definition 13. (Choquet [11], Calvo et al. [9]) The discrete Choquet integral with respect to a discrete fuzzy measure $\mu$ is the function $C^{\mu}: \mathbb{R}^{n} \longrightarrow \mathbb{R}$ given by

$$
C^{\mu}\left(a_{1}, \ldots, a_{n}\right)=\sum_{i=1}^{n} a_{(i)}\left[\mu\left(\left\{j \mid a_{j} \geqslant a_{(i)}\right\}\right)-\mu\left(\left\{j \mid a_{j} \geqslant a_{(i+1)}\right\}\right)\right],
$$

where $\boldsymbol{a}_{\nearrow}=\left(a_{(1)}, \ldots, a_{(n)}\right)$ is a non-decreasing permutation of $\boldsymbol{a}=\left(a_{1}, \ldots, a_{n}\right)$, and $a_{(n+1)}=\infty$ by convention.

Any discrete Choquet integral operator $C^{\mu}$ is compensative, i.e., for every $\mathbf{a}=\left\{a_{1}, \ldots, a_{n}\right\} \in[0,1]^{n}$,

$$
\min \left\{a_{1}, \ldots, a_{n}\right\} \leqslant C^{\mu}\left(a_{1}, \ldots, a_{n}\right) \leqslant \max \left\{a_{1}, \ldots, a_{n}\right\} .
$$

This means that we can restrict $C^{\mu}$ to $[0,1]^{n}$ and define $F^{\mu}:[0,1]^{n} \longrightarrow[0,1]$. In fact Marichal [27] argues that $C^{\mu}$ can be defined unambiguously on $[0,1]^{n}$, hence $F^{\mu}$ is also called a discrete Choquet integral. Now we are ready to introduce the following novel class of FCIFs:

Definition 14. Let $\mu$ be a discrete fuzzy measure and let $F^{\mu}$ be given by the restriction of Definition 13 to $[0,1]^{n}$. The Choquet-FCIF associated with $\mu$ is defined as $\boldsymbol{J}^{\mu}: \mathbf{M} \longrightarrow \mathrm{FS}(N)$ such that for each $M \in \mathbf{M}$,

$$
\boldsymbol{J}^{\mu}(M)(i)=F^{\mu}\left(M_{1 i}, \ldots, M_{n i}\right), \text { for each } i \in N .
$$

Properties of Choquet-FCIFs. In order to justify that Choquet-FCIFs perform well in our framework, we can import some properties from the discrete Choquet integral operator. Let us fix the discrete fuzzy measure $\mu$.

1. Any discrete Choquet integral operator $F^{\mu}$ is monotonic or increasing in each argument:

$$
F^{\mu}(\mathbf{a}) \geqslant F^{\mu}(\mathbf{b})
$$

whenever $\mathbf{a}, \mathbf{b} \in[0,1]^{n}$ and $\mathbf{a} \geqslant \mathbf{b}$, i.e., $a_{i} \geqslant b_{i}$ for each $i$. This produces the monotonicity property of Choquet-FCIFs, a natural extension of the usual monotonicity in Social Choice (cf., Samet and Schmeidler [32]). Formally, for each $i \in N$ :

$$
\mathbf{J}^{\mu}(M)(i) \leqslant \mathbf{J}^{\mu}\left(M^{\prime}\right)(i) \text { when } M_{j i} \leqslant M_{j i}^{\prime} \text { for each } j=1, \ldots, n .
$$

Monotonicity guarantees the following good features:

(a) For any given fuzzy profile, if the degrees of membership of individual $i$ are not smaller (across the members of the society) than the degrees of membership of individual $j$, then the degree of membership of $i$ is not smaller than the degree of membership of $j$. 
(b) If we study two fuzzy profiles and the degrees of membership of an individual are not smaller under the first profile (across the members of the society), then her degree of membership under the first profile is not smaller than her degree of membership under the second one.

2. The fact that Choquet integrals are compensative implies that the degree of membership of an individual is neither lower that her lowest evaluation nor higher than her highest evaluation. We call this property of Choquet-FCIFs compensativeness.

3. It is known that any discrete Choquet integral operator $F^{\mu}$ is idempotent: for every $a \in[0,1], F^{\mu}(a, \ldots, a)=a$.

Therefore if all persons in the society agree that the degree of membership of an individual is $a$ then it is socially accepted that $a$ is her degree of membership. Borrowing terminology from Social Choice, this is the unanimity property of Choquet-FCIFs. In particular, $\mathbf{J}^{\mu}(M)(i)=0$ if column $i$ of $M$ is constantly 0 , and $\mathbf{J}^{\mu}(M)(i)=1$ if column $i$ of $M$ is constantly 1 .

4. Any discrete Choquet integral operator $F^{\mu}$ is stable for admissible positive lineal transformations ${ }^{9}$

$$
F^{\mu}\left(r \cdot a_{1}+s, \ldots, r \cdot a_{n}+s\right)=r \cdot F^{\mu}\left(a_{1}, \ldots, a_{n}\right)+s,
$$

when $\mathbf{a}=\left\{a_{1}, \ldots, a_{n}\right\} \in[0,1]^{n}$, for $r>0$ and $s \in \mathbb{R}$ such that $r \cdot a_{j}+s \in[0,1]$ for each $j=1, \ldots, n$.

Hence Choquet-FCIFs inherit a property of stability for positive linear transformations. Formally, for each $i \in N$ :

$$
\mathbf{J}^{\mu}(M)(i)=r \cdot \mathbf{J}^{\mu}\left(M^{\prime}\right)(i)+s
$$

when $M_{j i}=r M_{j i}^{\prime}+s$ for each $j=1, \ldots, n, r>0, s \in \mathbb{R}$ with $M_{j i}, M_{j i}^{\prime} \in$ $[0,1]$ for each $j=1, \ldots, n$.

5. Any discrete Choquet integral operator $F^{\mu}$ is comonotonic additive:

$$
F^{\mu}\left(a_{1}+b_{1}, \ldots, a_{n}+b_{n}\right)=F^{\mu}\left(a_{1}, \ldots, a_{n}\right)+F^{\mu}\left(b_{1}, \ldots, b_{n}\right)
$$

when $\mathbf{a}, \mathbf{b} \in[0,1]^{n}$ are two comonotonic vectors. We recall that $\mathbf{a}, \mathbf{b} \in$ $[0,1]^{n}$ are comonotonic if there exists a permutation $\pi$ of $N$ such that $a_{\pi(1)} \leqslant a_{\pi(2)} \leqslant \ldots \leqslant a_{\pi(n)}$ and $b_{\pi(1)} \leqslant b_{\pi(2)} \leqslant \ldots \leqslant b_{\pi(n)}$.

This property ensures that Choquet-FCIFs are comonotonicity additive. Formally, for each $i \in N$ :

$$
\mathbf{J}^{\mu}\left(M+M^{\prime}\right)(i)=\mathbf{J}^{\mu}(M)(i)+\mathbf{J}^{\mu}\left(M^{\prime}\right)(i)
$$

when columns $M^{i}$ and $M^{\prime i}$ are comonotonic. Obviously, we say that $M^{i}$ and $M^{\prime i}$ are comonotonic when there is $\pi$, a permutation of $N$, such that

$$
M_{\pi(1) i} \leqslant M_{\pi(2) i} \leqslant \ldots \leqslant M_{\pi(n) i} \text { and } M_{\pi(1) i}^{\prime} \leqslant M_{\pi(2) i}^{\prime} \leqslant \ldots \leqslant M_{\pi(n) i}^{\prime}
$$

${ }^{9}$ This property is called homogeneity of degree 1 or ratio scale invariance when $s=0$ [25]. 
or equivalently, when

$$
\left(M_{j i}-M_{k i}\right)\left(M_{j i}^{\prime}-M_{k i}^{\prime}\right) \geqslant 0 \text { for each } j, k \in\{1, \ldots, n\} .
$$

6. It is well known that the discrete Choquet integral is uniquely defined by its values at the vertices of the unit cube $[0,1]^{n}$, i.e., at the points $\mathbf{a}=$ $\left(a_{1}, \ldots, a_{n}\right)$ such that $a_{i} \in\{0,1\}$ for each $i$. There are $2^{n}$ such points, the same as the number of values that determine the fuzzy measure $\mu$. From this property we deduce that $\mathbf{J}^{\mu}$ is completely determined by its restriction to $\mathcal{M}$, the collection of all crisp individual profiles.

In addition, the Choquet-FCIF verifies other interesting properties. For further use we emphasize the following one:

7. Independence: If $M$ and $M^{\prime}$ are two fuzzy profiles such that their respective columns $i$ coincide, then the degree of membership of agent $i$ to the group identity is the same under both profiles: $\mathbf{J}^{\mu}(M)(i)=\mathbf{J}^{\mu}\left(M^{\prime}\right)(i)$. ${ }^{10}$

Example 2. Consider the following fuzzy profile $M \in \mathbf{M}$ :

$$
M=\left(\begin{array}{lll}
0.18 & 0.10 & 0.4 \\
0.16 & 0.12 & 0.5 \\
0.10 & 0.18 & 0.4
\end{array}\right)
$$

We assume the following fuzzy measure: $\mu(\{1\})=\mu(\{2\})=0.45, \mu(\{3\})=0.3$, $\mu(\{1,2\})=0.5$, and $\mu(\{1,3\})=\mu(\{2,3\})=0.9$ (together with the compulsory $\mu(\varnothing)=0$ and $\mu(\{1,2,3\})=1)$. The fact $\mu(\{1\})+\mu(\{2\})>\mu(\{1,2\})$ means redundancy between criteria of agents 1 and 2 . The fact $\mu(\{1\})+\mu(\{3\})<$ $\mu(\{1,3\})$, resp., $\mu(\{2\})+\mu(\{3\})<\mu(\{2,3\})$, means synergism between criteria of agents 1 and 3, resp., of agents 2 and 3 .

Let us compute the degrees of membership of the agents to the group by the Choquet-FCIF associated with the aforementioned $\mu$. It is a matter of calculation to show that:

- The degree of membership of the first person is

$$
\mathbf{J}^{\mu}(M)(1)=0.1+0.06 \mu(\{1,2\})+0.02 \mu(\{1\})=0.139 .
$$

- The degree of membership of the second person is

$$
\mathbf{J}^{\mu}(M)(2)=0.1+0.02 \mu(\{2,3\})+0.06 \mu(\{3\})=0.136 .
$$

- The degree of membership of the third person is

$$
\mathbf{J}^{\mu}(M)(3)=0.4+0.1 \mu(\{2\})=0.445 \text {. }
$$

${ }^{10}$ In subsection 5.1 we refer to the traditional statement and interpretation of the properties of unanimity and independence (in the analysis of a remarkable impossibility theorem in the traditional framework). 


\subsection{The family of $O W A$-FCIFs.}

As a particular case of the discrete Choquet integral, we can utilize OWAs as averaging aggregation functions (cf., Yager [38]) in order to define a particular subclass of the Choquet-FCIF called OWA-FCIF. OWA operators allow us to calculate aggregate values by weighting the supplied values in relation to their relative ordering. OWA operators are special cases of Choquet integrals with respect to symmetric fuzzy measures, or in other words, symmetric Choquet integral operators (cf., Calvo, Mayor and Mesiar [9]). Hence we now recall the concept of an OWA operator:

Definition 15 (Yager [38]). Let $\boldsymbol{w}=\left(w_{1}, \ldots, w_{n}\right) \in[0,1]^{n}$ be a weighting vector such that $\sum_{i=1}^{n} w_{i}=1$. The ordered weighted averaging (OWA) operator associated with $\boldsymbol{w}$ is the function $F^{\boldsymbol{w}}: \mathbb{R}^{n} \longrightarrow \mathbb{R}$ defined by

$$
F^{w}\left(a_{1}, \ldots, a_{n}\right)=\sum_{i=1}^{n} w_{i} b_{i} \text { for each }\left(a_{1}, \ldots, a_{n}\right) \in \mathbb{R}^{n}
$$

where $b_{i}$ is the $i$-th largest element in the collection of (possibly repeated) values $\left\{a_{1}, \ldots, a_{n}\right\}$.

We are ready to define the notion of $O W A$-FCIF.

Definition 16. Let us fix $\boldsymbol{w}=\left(w_{1}, \ldots, w_{n}\right) \in[0,1]^{n}$ weighting vector with $\sum_{i=1}^{n} w_{i}=1$, and let $F^{\boldsymbol{w}}$ be given by Definition 15. The OWA-FCIF associated with $\boldsymbol{w}$ is defined as $\boldsymbol{J}^{w}: \mathbf{M} \longrightarrow \mathrm{FS}(N)$ such that for each $M \in \mathbf{M}$,

$$
\boldsymbol{J}^{w}(M)(i)=F^{w}\left(M_{1 i}, \ldots, M_{n i}\right), \text { for each } i \in N \text {. }
$$

Of course, many noteworthy examples arise from concrete specifications of the weight vector. The case $\boldsymbol{w}=\left(\frac{1}{n}, \ldots, \frac{1}{n}\right)$ yields the average mean FCIF: see Figure 1. This fact replicates the property that the average mean operator is an OWA operator with equal weights. The case $\boldsymbol{w}=(0,0, \ldots, 1)$ yields the conjunctive FCIF that evaluates the degree of membership of each agent by his or her smallest qualification by the group. The case $\boldsymbol{w}=(1,0, \ldots, 0)$ evaluates the degree of membership of each agent by his or her highest qualification by the group thus it captures a very permissive view of group identity by the benevolent FCIF. Nevertheless, neither the liberal nor the dictatorial FCIFs are particular cases of $O W A$-FCIFs. Table 2 summarizes these peculiarities.

Properties of $\boldsymbol{O W A}$-FCIFs. Of course, some properties of $O W A$-FCIFs are inherited from properties of Choquet-FCIFs (e.g., monotonicity, compensativeness, idempotency, et cetera). But we can also import relevant properties of the OWA operator in order to assure that $O W A$-FCIFs have certain distinctive behavioral attributes in our setting: 


\begin{tabular}{ll} 
Weight vector & Decision Rule \\
\hline $\boldsymbol{w}=\left(\frac{1}{n}, \ldots, \frac{1}{n}\right)$ & Average mean $\mathrm{FCIF}$ \\
$\boldsymbol{w}=(0,0, \ldots, 1)$ & Conjunctive FCIF \\
$\boldsymbol{w}=(1,0, \ldots, 0)$ & Benevolent $\mathrm{FCIF}$ \\
\hline
\end{tabular}

Table 2: Summary table of particular examples of $O W A$-FCIFs according to their weight vectors.

1. Any OWA operator $F^{w}$ is $\sigma$-homogeneous of degree 1:

$$
F^{w}(\lambda \mathbf{a})=\lambda F^{w}(\mathbf{b})
$$

whenever vector $\mathbf{b}$ is a permutation of vector $\mathbf{a}$ and $\lambda>0$. This implies that the degree of membership of an individual whose evaluations are e.g., the result of systematically halving the evaluations of another individual (in any order), is half the degree of membership of the latter person. We call this property of $O W A$-FCIFs $\sigma$-homogeneity of degree 1 . It implies homogeneity of degree 1 when $\sigma$ is the identity.

2. Due to $\sigma$-homogeneity of degree 1 , the opinions of all agents are equally important. Borrowing terminology from Social Choice, this is the anonymity property: $\mathbf{J}^{\boldsymbol{w}}(M)(i)$ is invariant by permutations of the evaluations in column $i$ of $M$.

In addition, the $O W A$-FCIF verifies independence as well.

Remark 1. We address the reader to Calvo and Beliakov [8] for exhaustive information on the problem of the identification of weights in aggregation operators.

Example 3. Let us revisit the fuzzy profile $M^{\prime} \in \mathbf{M}$ defined in Example 1. In order to apply Definition 16, we use the following rearrangements of the columns of $M^{\prime}$ :

$-\mathbf{b}_{1}=(0.9,0.8,0.7,0.6,0.6,0.6)$ which is used to produce

$$
\mathbf{J}^{w}\left(M^{\prime}\right)(1)=F^{w}\left(M_{11}^{\prime}, \ldots, M_{n 1}^{\prime}\right)
$$

$-\mathbf{b}_{2}=(1,0.9,0.9,0.8,0.7,0.7)$, which is used to produce

$$
\mathbf{J}^{\boldsymbol{w}}\left(M^{\prime}\right)(2)=F^{\boldsymbol{w}}\left(M_{12}^{\prime}, \ldots, M_{n 2}^{\prime}\right) .
$$

$-\mathbf{b}_{3}=(0.3,0.2,0.2,0,0,0)$, which is used to produce

$$
\mathbf{J}^{w}\left(M^{\prime}\right)(3)=F^{w}\left(M_{13}^{\prime}, \ldots, M_{n 3}^{\prime}\right)
$$

$-\mathbf{b}_{4}=(0.6,0.5,0.5,0.4,0.4,0.3)$, which is used to produce

$$
\mathbf{J}^{\boldsymbol{w}}\left(M^{\prime}\right)(4)=F^{\boldsymbol{w}}\left(M_{14}^{\prime}, \ldots, M_{n 4}^{\prime}\right)
$$


$-\mathbf{b}_{5}=(0.8,0.7,0.6,0.5,0.5,0.5)$, which is used to produce

$$
\mathbf{J}^{\boldsymbol{w}}\left(M^{\prime}\right)(5)=F^{\boldsymbol{w}}\left(M_{15}^{\prime}, \ldots, M_{n 5}^{\prime}\right) .
$$

- $\mathbf{b}_{6}=(0.5,0.4,0.3,0.3,0.2,0.1)$, which is used to produce

$$
\mathbf{J}^{\boldsymbol{w}}\left(M^{\prime}\right)(6)=F^{w}\left(M_{16}^{\prime}, \ldots, M_{n 6}^{\prime}\right) .
$$

Now it is simple to compute the degrees of membership of the agents to the group for any $O W A$-FCIF, i.e., for any possible weight vector $\boldsymbol{w}$. Thus for example:

- The average mean FCIF, $\mathbf{J}_{a}$, uses $\boldsymbol{w}=\left(\frac{1}{6}, \ldots, \frac{1}{6}\right)$ and the degree of membership of each agent is shown in Table 3 .

Table 3: OWA-FCIF degrees of membership with $\boldsymbol{w}=\left(\frac{1}{6}, \ldots, \frac{1}{6}\right)$.

$$
\begin{array}{lllllll} 
& 1 & 2 & 3 & 4 & 5 & 6 \\
\hline \mathbf{J}_{a}\left(M^{\prime}\right)(i) & 0.70 & 0.83 & 0.12 & 0.45 & 0.6 & 0.3 \\
\hline
\end{array}
$$

- When $\boldsymbol{w}=(0,0.3,0.3,0.2,0.2,0)$ the degree of membership of each agent is given in Table 4.

Table 4: OWA-FCIF degrees of membership with $\boldsymbol{w}=(0,0.3,0.3,0.2,0.2,0)$.

\begin{tabular}{lllllll} 
& 1 & 2 & 3 & 4 & 5 & 6 \\
\hline $\mathbf{J}^{\boldsymbol{w}}\left(M^{\prime}\right)(i)$ & 0.69 & 0.84 & 0.08 & 0.46 & 0.59 & 0.31 \\
\hline
\end{tabular}

\subsection{The family of $W O W A-F C I F s$.}

In an OWA operator, weights establish the importance of a value in relation to other values. This is done regardless of the agent or source that emitted the value. However in many group decision mechanisms, agents play different roles and it is sensible to propose procedures that allow for these disparities. In our context, to that purpose we can use WOWA operators which are not necessarily invariant to permutations of the membership degrees attached (to a fixed person). By the recourse to a WOWA operator we can give personalized importance to each member's opinions. This requires the introduction of an additional input, namely, the weights $\mathbf{p}$ that correspond to the agents (in addition to the weights $\boldsymbol{w}$ that graduate the importance of the evaluations in relation to other evaluations). 
Definition 17. Let $\mathbf{p}=\left(p_{1}, \ldots, p_{n}\right) \in[0,1]^{n}$ and $\boldsymbol{w}=\left(w_{1}, \ldots, w_{n}\right) \in[0,1]^{n}$ be weighting vectors with $\sum_{i=1}^{n} p_{i}=1, \sum_{i=1}^{n} w_{i}=1$. The WOWA-FCIF associated with $\mathbf{p}$ and $\boldsymbol{w}$ is defined as $\mathbf{J}^{\boldsymbol{w}, \mathbf{p}}: \mathbf{M} \longrightarrow \mathrm{FS}(N)$ such that for each $M \in \mathbf{M}$,

$$
\mathbf{J}^{w, \mathbf{p}}(M)(i)=\mathbf{J}^{w, \mathbf{p}}\left(M_{\sigma(1) i}, \ldots, M_{\sigma(n) i}\right)=\sum_{j=1}^{n} \lambda_{j} M_{\sigma(j) i} \text { for each } i \in N
$$

where $\sigma:\{1, \ldots, n\} \rightarrow\{1, \ldots, n\}$ is a permutation function such that $M_{\sigma(1) i} \geqslant M_{\sigma(2) i} \geqslant \ldots \geqslant M_{\sigma(n) i}$ and the weights $\lambda_{i}$ are defined as

$$
\lambda_{i}=f\left(\sum_{j=1}^{i} p_{\sigma(j)}\right)-f\left(\sum_{j=1}^{i-1} p_{\sigma(j)}\right)
$$

where $f$ is a non-decreasing function that interpolates the points $\left(\frac{i}{n}, \sum_{j=1}^{i} w_{j}\right)$ together with the point $(0,0)$, with the proviso that $f$ is the identity when the identity interpolates these points.

Properties of WOWA-FCIFs. We can also import properties of the WOWA operator to assure that the WOWA-FCIF has certain behavioral attributes in our setting.

1. Suppose $\mathbf{p}=\left(\frac{1}{n}, \ldots, \frac{1}{n}\right)$. Then the WOWA-FCIF is an $O W A$-FCIF associated with $\boldsymbol{w}$ (because Torra [34, Proposition 5] establishes the corresponding property for WOWA operators: see Figure 1). Therefore, when in addition $\boldsymbol{w}=\left(\frac{1}{n}, \ldots, \frac{1}{n}\right)$ we obtain the average mean FCIF (AM-FCIF). If $\boldsymbol{w}=$ $(0, \ldots, 0,1)$, we obtain the conjunctive FCIF. If $\boldsymbol{w}=(1,0, \ldots, 0)$, we obtain the benevolent FCIF.

2. Suppose $\boldsymbol{w}=\left(\frac{1}{n}, \ldots, \frac{1}{n}\right)$. Then the WOWA-FCIF is called weighted mean FCIF associated with the weighting vector $\mathbf{p}$ (WM-FCIF). Torra [34, Proposition 4] establishes the corresponding property for WOWA operators: see Figure 1. Of course, when in addition $\mathbf{p}=\left(\frac{1}{n}, \ldots, \frac{1}{n}\right)$ we obtain the average mean FCIF.

3. When $\mathbf{p}$ verifies $p_{i}=1, p_{j}=0$ for each $j \neq i$, the WOWA-FCIF is the dictatorial FCIF by agent $i$ (because Torra [34, Proposition 6 (5)] establishes the corresponding property for WOWA operators).

4. Any WOWA operator $F^{w, \mathbf{p}}$ is homogeneous of degree 1 (cf., Llamazares $[25])$ :

$$
F^{w, \mathbf{p}}(\lambda \mathbf{a})=\lambda F^{w, \mathbf{p}}(\mathbf{a})
$$

We call the induced property of WOWA-FCIFs homogeneity of degree 1: This implies that the degree of membership of an individual whose evaluations are e.g., the result of systematically halving the evaluations of another individual (in any order), is half the degree of membership of the latter person. 
5. Any WOWA operator is monotonic (Torra [34, Proposition 6 (4)]). This yields the monotonicity property of WOWA-FCIFs, a natural extension of the monotonicity property of $O W A$-FCIFs whose interpretation has been given above.

6. Any WOWA operator is compensative (Torra [34, Proposition 6 (1)]). This property assures that WOWA-FCIFs verify compensativeness defined as in the case of $O W A$-FCIFs. Therefore, WOWA-FCIFs verify the unanimity property that extends the corresponding definition for $O W A$-FCIFs.

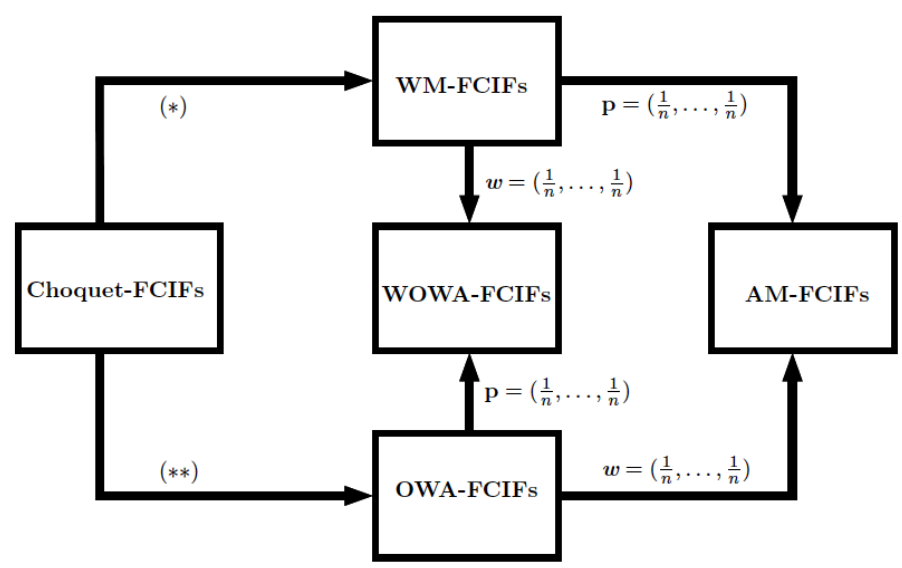

Fig. 1: Relationships among Choquet-FCIFs, WOWA-FCIFs, OWA-FCIFs, WM-FCIFs and AM-FCIFs. Grabisch et al. [21, Proposition 5.50] permits to identify the precise conditions that guarantee $(*)$ and $(* *)$.

Remark 2. Torra and Lv [36] introduce a definition that emphasizes the role of the interpolation method used in an OWA operator. They define WOWAconsistent interpolation methods and study to which extent the selected interpolation method influences the result of the WOWA operator. Furthermore, already Torra [35] explained that it is also possible to determine WOWAs from a quantifier and the weighting vector $\mathbf{p}=\left(p_{1}, \ldots, p_{n}\right) \in[0,1]^{n}$. Therefore we could also define a WOWA-FCIF associated with $\mathbf{p}$ and the quantifier $Q$ (Yager [39]). We skip this trivial modification of Definition 17.

Example 4. Consider the following fuzzy profile $M^{\prime \prime} \in \mathbf{M}$ :

$$
M^{\prime \prime}=\left(\begin{array}{rrrrr}
1 & 0 & 0.4 & 0.7 & 0.5 \\
0.8 & 0 & 0.6 & 0.5 & 0.3 \\
0.7 & 0.2 & 0.4 & 0.5 & 0.4 \\
0.9 & 0.2 & 0.3 & 0.8 & 0.3 \\
0.7 & 0.3 & 0.5 & 0.5 & 0.1
\end{array}\right)
$$


$M^{\prime \prime}$ is the fuzzy subprofile of $M^{\prime}$ defined in Example 1 that disregards the first member of the society.

We assume that the importance of the opinions of the current members of the society are weighed by $\mathbf{p}=(0.3,0.2,0.2,0.2,0.1)$. We also use $\boldsymbol{w}=\left(0, \frac{1}{3}, \frac{1}{3}, \frac{1}{3}, 0\right)$ under which central assessments are equally valued and extreme values are discarded. We follow the exposition in Llamazares [25, Example 1] and choose the following function in order to interpolate the points $(0,0),(0.2,0),(0.4,1 / 3)$, $(0.6,2 / 3),(0.8,1)$, and $(1,1)$ :

$$
f(x)=\left\{\begin{array}{l}
0, \text { if } x \leq 0.2 \\
\frac{5}{3} x-\frac{1}{3}, \quad \text { if } 0.2<x<0.8 \\
1, \text { if } \quad x \geq 0.8
\end{array}\right.
$$

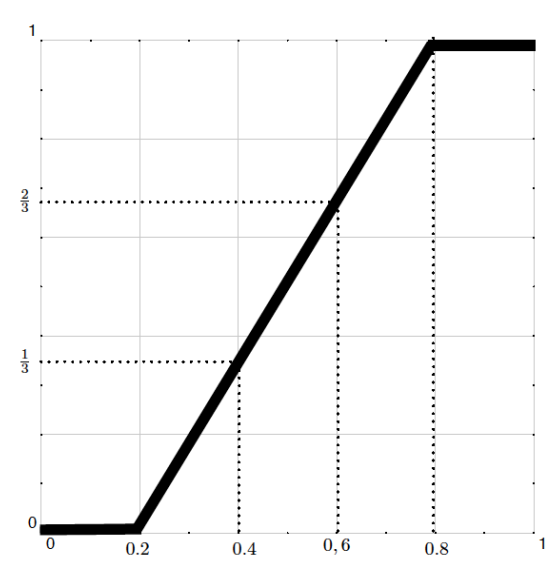

Fig. 2: A non-decreasing function associated with $\boldsymbol{w}=\left(0, \frac{1}{3}, \frac{1}{3}, \frac{1}{3}, 0\right)$.

Now in order to evaluate the degree of membership of each individual to the collective identity, we proceed as follows.

- Member 1 . We use the following rearrangement of $M^{\prime \prime(1)}$, the first column of $M^{\prime \prime}$ :

$$
\left(M_{\sigma(1) 1}, M_{\sigma(2) 1}, M_{\sigma(3) 1}, M_{\sigma(4) 1}, M_{\sigma(5) 1}\right)=(1,0.9,0.8,0.7,0.7)
$$

whose associated permutation of the $\mathbf{p}$ vector is $\mathbf{p}_{1}=\mathbf{p}$. Now $\lambda_{1}=\lambda_{4}=\frac{1}{6}$, $\lambda_{2}=\frac{1}{3}=\lambda_{3}, \lambda_{5}=0$. The degree of membership of this person is

$$
\mathbf{J}^{w, \mathbf{p}}\left(M^{\prime \prime}\right)(1)=\frac{1}{6} \cdot 1+\frac{1}{3} \cdot 0.9+\frac{1}{3} \cdot 0.8+\frac{1}{6} \cdot 0.7+0=0.85
$$


- Member 2. We use the following rearrangement of $M^{\prime \prime(2)}$ :

$$
\left(M_{\sigma(1) 2}, M_{\sigma(2) 2}, M_{\sigma(3) 2}, M_{\sigma(4) 2}, M_{\sigma(5) 2}\right)=(0.3,0.2,0.2,0,0)
$$

whose associated permutation of the $\mathbf{p}$ vector is $\mathbf{p}_{2}=(0.1,0.2,0.2,0.2,0.3)$. Now $\lambda_{1}=0, \lambda_{2}=\frac{1}{6}, \lambda_{3}=\frac{1}{3}=\lambda_{4}, \lambda_{5}=\frac{1}{6}$. The degree of membership of this person is

$$
\mathbf{J}^{w, \mathbf{p}}\left(M^{\prime \prime}\right)(2)=0.3 \cdot 0+\frac{1}{6} \cdot 0.2+\frac{1}{3} \cdot 0.2+\frac{1}{3} \cdot 0+\frac{1}{3} \cdot 0=0.1
$$

- Member 3. We use the following rearrangement of $M^{\prime \prime(3)}$ :

$$
\left(M_{\sigma(1) 3}, M_{\sigma(2) 3}, M_{\sigma(3) 3}, M_{\sigma(4) 3}, M_{\sigma(5) 3}\right)=(0.6,0.5,0.4,0.4,0.3)
$$

whose associated permutation of the $\mathbf{p}$ vector is $\mathbf{p}_{3}=(0.2,0.1,0.2,0.3,0.2)$. Now $\lambda_{1}=0, \lambda_{2}=\frac{1}{6}, \lambda_{3}=\frac{1}{3}, \lambda_{4}=\frac{1}{2}, \lambda_{5}=0$. The degree of membership of this person is

$$
\mathbf{J}^{\boldsymbol{w}, \mathbf{p}}\left(M^{\prime \prime}\right)(3)=0.6 \cdot 0+\frac{1}{6} \cdot 0.5+\frac{1}{3} \cdot 0.4+\frac{1}{2} \cdot 0.4+0 \cdot 0.3 \approx 0.417
$$

- Member 4. We use the following rearrangement of $M^{\prime \prime(4)}$ :

$$
\left(M_{\sigma(1) 4}, M_{\sigma(2) 4}, M_{\sigma(3) 4}, M_{\sigma(4) 4}, M_{\sigma(5) 4}\right)=(0.8,0.7,0.5,0.5,0.5)
$$

whose associated permutation of the $\mathbf{p}$ vector is $\mathbf{p}_{4}=(0.2,0.3,0.2,0.2,0.1)$. Now $\lambda_{1}=0, \lambda_{2}=\frac{1}{2}, \lambda_{3}=\frac{1}{3}, \lambda_{4}=\frac{1}{6}, \lambda_{5}=0$. The degree of membership of this person is

$$
\mathbf{J}^{w, \mathbf{p}}\left(M^{\prime \prime}\right)(4)=0.8 \cdot 0+\frac{1}{2} \cdot 0.7+\frac{1}{3} \cdot 0.5+\frac{1}{6} \cdot 0.5+0 \cdot 0.5=0.6
$$

- Member 5. We use the following rearrangement of $M^{\prime \prime(5)}$ :

$$
\left(M_{\sigma(1) 5}, M_{\sigma(2) 5}, M_{\sigma(3) 5}, M_{\sigma(4) 5}, M_{\sigma(5) 5}\right)=(0.5,0.4,0.3,0.3,0.1)
$$

whose associated permutation of the $\mathbf{p}$ vector is $\mathbf{p}_{5}=(0.3,0.2,0.2,0.2,0.1)$. Now $\lambda_{1}=\frac{1}{6}, \lambda_{2}=\frac{1}{3}, \lambda_{3}=\frac{1}{3}, \lambda_{4}=\frac{1}{6}, \lambda_{5}=0$. The degree of membership of this person is

$$
\mathbf{J}^{w, \mathbf{p}}\left(M^{\prime \prime}\right)(5)=\frac{1}{6} \cdot 0.5+\frac{1}{3} \cdot 0.4+\frac{1}{3} \cdot 0.3+\frac{1}{6} \cdot 0.3+0 \cdot 0.1 \approx 0.367
$$

Remark 3. Damiani et al. [12] give another interesting use of a linear interpolation in their analysis of a WOWA-based aggregation technique on individual trust values on metadata assertions. 


\section{Conclusions and future research}

We have reconsidered the problem of self-selection of individuals in a group with respect to a given attribute. Since these attributes are usually vague or imprecise, it seems just natural to approach the problem from a fuzzy rather than a crisp or categorical position. Miller [28, Subsection 3.1] tacitly recognized this need when he claimed the following as a desideratum: "A possible extension to the group identification model would be to weaken the assumption that opinions about qualification are binary by replacing the domain of possible opinions from $\{0,1\}$ to an arbitrary totally ordered set $D$ containing minimal and maximal elements 0 and 1." We are not aware of any contribution that explores this route in its generality. Here we go beyond Miller's goal by defining the new problem of collective identification in a framework where the agents express their judgements and are ultimately qualified through partial memberships. Other weaker frameworks are possible, inclusive of the standard case where both the opinions and the resulting memberships are crisp. Our main new tool of analysis is the notion of FCIFs. The existing literature provided some focal rules that can be naturally extended to the new framework. But the richer structure that we have allowed in our model permits to be more flexible in the assessments, which reduces losses of information. Particularly interesting are Choquet- and WOWA-FCIFs, which include OWA-FCIFs. Not only the membership assessments received can be weighted in relation to other evaluations, but also the opinions of the agents can be treated differently (e.g., to recognize seniorship) or corrected from interactions among opinions (e.g., to counteract lobbies).

In order to endorse the advantages of our model, we point out at two additional developments. In subsection 5.1 we see that its versatility permits to avoid a well-known impossibility in the traditional approach to endogenous selection. Then subsection 5.2 explores the possibility of producing characterizations of relevant classes of FCIFs. In subsection 5.3 we give some final remarks.

\subsection{A positive escape to Kasher and Rubinstein's impossibility theorem}

In their seminal contribution, Kasher and Rubinstein [24] proved the following result:

Theorem 1. The only $C I F^{*}$ 's that verify consensus and independence are the dictatorships.

According to their Section 5, a CIF* is a function that assigns a proper subset of $N$ to every profile of proper subsets of $N$. I.e., it is assumed that there is a consensus in the group that the self-selected subgroup of qualified members must be a proper subset of $N$. The consensus property captures the meaning of our unanimity property (cf., subsection 4.1). It means that if there is an agreement among all individuals that a certain individual qualifies (resp., does not qualify) as a member, then the CIF* determines that this member belongs (resp., does not 
belong) to the endogenously selected group. And in their words, independence means that whether $i$ qualifies or not depends only on how individuals qualify $i$. Thus it coincides with the meaning that we attach to this term.

In order to show that the fuzzy view of the problem permits to escape this astringent result, we observe the following. For any weight vector with nonzero weights throughout, let us restrict the associated WMFCIF to the domain $\mathbf{M}-\{M(0), M(1)\}$. Hence we only exclude the profile that evaluates everyone with a 0 , and the profile that evaluates everyone with a 1 , from the domain. It is easy to check that our restricted WMFCIF verifies consensus and independence, and it neither qualifies everyone with full membership nor with null membership. Thus it provides a fuzzy restatement of a $\mathrm{CIF}^{*}$ on a less reduced domain whereas it is clearly non-dictatorial.

\subsection{On the characterization of classes of FCIFs}

Ir order to favor prospective uses in Social Choice it seems interesting to produce axiomatic characterizations of the most important new definitions of FCIFs. This follows the tradition of the works mentioned above in the extended approach to group identification (cf., [16], [22], [24], [28], [32], [33], among others). To that purpose the researcher could avail herself of either existing or novel characterizations of aggregation operators to the framework of our contribution.

Let us exemplify this technique with a characterization of the ChoquetFCIFs. We can take advantage of the characterization of the Choquet integral defined on $[0,1]^{n}$ (cf., Marichal [27, Theorem 3.1]). Marichal proves that it is the only aggregation function from $[0,1]^{n}$ to $\mathbb{R}$ that is stable for the admissible positive linear transformations (SPL), increasing in each opinion (IN) and comonotonic additive (CoAdd). This result easily produces the desired characterization in terms of the following adapted axiomatics:

Theorem 2. An FCIF $\boldsymbol{J}: \mathbf{M} \longrightarrow \mathrm{FS}(N)$ is a Choquet-FCIF if and only if it verifies Independence, SSPL, SIN and SCoAdd.

The new properties that we use in this characterization are translations of the aforementioned properties from [27, Theorem $3.1(i)]$ to our statement, in the following terms.

Definition 18. An FCIF $\boldsymbol{J}: \mathbf{M} \longrightarrow \mathrm{FS}(N)$ verifies:

1. Social stability for the admissible positive linear transformations (SSPL) if for each $i \in N$ :

$$
\boldsymbol{J}(M)(i)=r \cdot \boldsymbol{J}\left(M^{\prime}\right)(i)+s
$$

when $M_{j i}=r M_{j i}^{\prime}+s$ for each $j=1, \ldots, n, r>0, s \in \mathbb{R}$ with $M_{j i}, M_{j i}^{\prime} \in$ $[0,1]$ for each $j=1, \ldots, n$.

2. Socially monotonic or increasing in each opinion (SIN) if for each $i \in N$ :

$$
\boldsymbol{J}(M)(i) \leqslant \boldsymbol{J}\left(M^{\prime}\right)(i) \text { when } M_{j i} \leqslant M_{j i}^{\prime} \text { for each } j=1, \ldots, n .
$$


3. Socially comonotonic additive (SCoAdd) if for each $i \in N$ :

$$
\boldsymbol{J}\left(M+M^{\prime}\right)(i)=\boldsymbol{J}(M)(i)+\boldsymbol{J}\left(M^{\prime}\right)(i)
$$

when columns $M^{i}$ and $M^{\prime i}$ are comonotonic. We say that $M^{i}$ and $M^{\prime i}$ are comonotonic when there is $\pi$, a permutation of $N$, such that

$$
M_{\pi(1) i} \leqslant M_{\pi(2) i} \leqslant \ldots \leqslant M_{\pi(n) i} \text { and } M_{\pi(1) i}^{\prime} \leqslant M_{\pi(2) i}^{\prime} \leqslant \ldots \leqslant M_{\pi(n) i}^{\prime}
$$

or equivalently, when

$$
\left(M_{j i}-M_{k i}\right)\left(M_{j i}^{\prime}-M_{k i}^{\prime}\right) \geqslant 0 \text { for each } i, j \in\{1, \ldots, n\} .
$$

It is crucial to observe that Independence must be introduced in order to grant that the $n$ capacities obtained by invoking [27, Theorem 3.1] coincide accross agents.

We do not intend to make an extensive analysis of this topic. The interested reader can easily adapt other sets of axioms that jointly characterize the Choquet integral, e.g., SPL, IN, and BOM or bisymmetric for orderable matrices: cf., [27, Theorem 3.1 (ii)].

With respect to other classes of FCIFs, let us mention that the literature has provided characterizations of the $O W A$ operator defined on $\mathbb{R}^{n}$ (cf., Grabisch et al. [21, Theorem 5.46]). An adapted characterization to $[0,1]^{n}$ would eventually produce a characterization of $O W A$-FCIFs by following the technique above. Obviously, this technique also applies to any other related aggregation operator.

\subsection{Questions for future research}

The issue of how identity affects economic outcomes has been attempted in Akerlof and Kranton [1] from a different perspective. Their purpose is to incorporate the psychology and sociology of identity into the study. Nevertheless the formal approach to which we adhere seems not to be linked to subsequent economic analyses in the literature. Hence a challenge (in either crisp or fuzzy statements of the collective identity problem) is its implementation into economic environments.

Another line of inspection is the investigation of procedures that produce crisp qualifications from fuzzy profiles (in the sense of subsection 3.3) on the basis of FCIFs. For such procedures it would be interesting to know which properties can be transferred from the original FCIF to the new procedure with restricted codomain $\mathcal{P}(N)$.

Relatedly, it seems plausible to introduce the fuzzy techniques into the analysis of group identification with $c$ prefixed classes or indetermined number of classes where the names of the groups matter (cf., Subsection 2.1 for the crisp motivation).

We expect to conduct further investigations of these issues in the future. 


\section{Acknowledgment}

The authors have benefitted from constructive comments by two anonymous reviewers and Radko Mesiar (Area Editor), Salvador Barberà, Carmen Beviá, Luis Corchón, Susana Díaz, Bonifacio Llamazares, Susana Montes, and the participants in a seminar presentation at University of Oviedo, the IV Jornadas de Trabajo sobre Sistemas de Votación, and the $40^{\text {th }}$ Simposio de la Asociación Española de Economía. Financial support by the Spanish Ministerio de Ciencia e Innovación under Project ECO2012-32178 (R. de Andrés Calle) is gratefully acknowledged.

\section{References}

1. G. A. Akerlof and R. E. Kranton. Economics and identity. The Quarterly Journal of Economics, 115(3):715-753, 2000.

2. K. Arrow. Social choice and individual values. Wiley, New York, 1951.

3. M. A. Ballester and J.L. García-Lapresta. A model of elitist qualification. Group Decision and Negotiation, 17:497-513, 2008.

4. M. A. Ballester and J.L. García-Lapresta. A recursive group decision-making procedure for choosing qualified individuals. International Journal of Intelligent Systems, 24(8):889-901, 2009.

5. C. R. Barrett. Fuzzy preferences and choice: A further progress report. Fuzzy Sets and Systems, 37(2):261-262, 1990.

6. C. R. Barrett, Prasanta K. Pattanaik, and M. Salles. On choosing rationally when preferences are fuzzy. Fuzzy Sets and Systems, 34(2):197-212, 1990.

7. A. Billot. How liberalism kills democracy or Sen's theorem revisited. Public Choice, 116:247-270, 2003.

8. T. Calvo and G. Beliakov. Identification of weights in aggregation operators. In Humberto Bustince, Francisco Herrera, and Javier Montero, editors, Fuzzy Sets and Their Extensions: Representation, Aggregation and Models, volume 220 of Studies in Fuzziness and Soft Computing, pages 145-162. Springer Berlin Heidelberg, 2008.

9. T. Calvo, A. Kolesárová, M. Komorníková, and R. Mesiar. Aggregation operators: Basic concepts, issues and properties. In Tomasa Calvo, Gaspar Mayor, and Radko Mesiar, editors, Aggregation Operators: New Trends and Applications, volume 97 of Studies in Fuzziness and Soft Computing, pages 3-104. Springer Berlin Heidelberg, 2002.

10. M. Çengelci and M.R. Sanver. Simple collective identity functions. Theory and Decision, 68(4):417-443, 2010.

11. G. Choquet. Theory of capacities. Annales de l'Institut Fourier, 5:131-295, 1954.

12. E. Damiani, S. De Capitani di Vimercati, P. Samarati, and M. Viviani. A WOWAbased aggregation technique on trust values connected to metadata. Electronic Notes in Theoretical Computer Science, 157(3):131-142, 2006. Proceedings of the First International Workshop on Security and Trust Management (STM 2005) Proceedings of the 1st International Workshop on Security and Trust Management.

13. R. de Andrés Calle, J. L. García-Lapresta, and L. Martínez. A multi-granular linguistic model for management decision-making in performance appraisal. Soft Computing, 14:21-34, 2010. 
14. D. Dimitrov. The Social Choice approach to group identification. In Enrique Herrera-Viedma et al., editors, Consensual Processes, volume 267 of Studies in Fuzziness and Soft Computing, pages 123-134. Springer Berlin Heidelberg, 2011.

15. D. Dimitrov and C. Puppe. Non-bossy social classification. Mathematical Social Sciences, 62(3):162-165, 2011.

16. D. Dimitrov, S.C. Sung, and Y. Xu. Procedural group identification. Mathematical Social Sciences, 54:137-146, 2007.

17. D. Dubois and J.-L. Koning. Social choice axioms for fuzzy set aggregation. Fuzzy Sets and Systems, 43(3):257-274, 1991.

18. M. Grabisch. The application of fuzzy integrals in multicriteria decision making. European Journal of Operational Research, 89(3):445-456, 1996.

19. M. Grabisch, I. Kojadinovic, and P. Meyer. A review of methods for capacity identification in Choquet integral based multi-attribute utility theory: Applications of the Kappalab R package. European Journal of Operational Research, 186(2):766785,2008 .

20. M. Grabisch and C. Labreuche. A decade of application of the Choquet and Sugeno integrals in multi-criteria decision aid. 4OR, 6(1):1-44, 2008.

21. M. Grabisch, J.-L. Marichal, R. Mesiar, and E. Pap. Aggregation Functions. Cambridge University Press, Cambridge, 2009.

22. N. Houy. "I want to be a J!": Liberalism in group identification problems. Mathematical Social Sciences, 54:59-70, 2007.

23. A. Kasher. Jewish collective identity. Temple University Press, 1993.

24. A. Kasher and A. Rubinstein. On the question 'Who is a J?'. Logique \& Analyse, 160:385-395, 1997.

25. B. Llamazares. An analysis of some functions that generalizes weighted means and OWA operators. International Journal of Intelligent Systems, 28(4):380-393, 2013.

26. J.-L. Marichal. An axiomatic approach of the discrete Choquet integral as a tool to aggregate interacting criteria. Fuzzy Systems, IEEE Transactions on, 8(6):800-807, 2000.

27. J.-L. Marichal. On Choquet and Sugeno integrals as aggregation functions. In M. Grabisch, T. Murofushi, and M. Sugeno, editors, Fuzzy Measures and Integrals. Theory and Applications, pages 247-272. Physica Verlag, Heidelberg, 2000.

28. A.D. Miller. Group identification. Games and Economic Behavior, 63:188-202, 2008.

29. J. Montero. Rational aggregation rules. Fuzzy Sets and Systems, 62(3):267-276, 1994.

30. J. Rawls. A Theory of Justice. Harvard University Press, 1971.

31. A. Rubinstein and P. C. Fishburn. Algebraic aggregation theory. Journal of Economic Theory, 38(1):63-77, 1986.

32. D. Samet and D. Schmeidler. Between liberalism and democracy. Journal of Economic Theory, 110:213-233, 2003.

33. A. Saporiti. A proof for 'Who is a J' impossibility theorem. Economics Bulletin, 32:494-501, 2012.

34. V. Torra. The weighted OWA operator. International Journal of Intelligent Systems, 12(2):153-166, 1997.

35. V. Torra. The WOWA operator and the interpolation function $\mathrm{W}^{*}$ : Chen and Otto's interpolation method revisited. Fuzzy Sets and Systems, 113(3):389-396, 2000 .

36. V. Torra and Z. Lv. On the WOWA operator and its interpolation function. International Journal of Intelligent Systems, 24(10):1039-1056, 2009. 
37. V. Torra and Y. Narukawa. Modeling decisions: Information fusion and aggregation operators. Springer, 2007.

38. R. R. Yager. On ordered weighted averaging aggregation operators in multicriteria decision making. IEEE Trans. on Systems, Man and Cybernetics, 18:183-190, 1988.

39. R. R. Yager. Families of OWA operators. Fuzzy Sets and Systems, 59(2):125-148, 1993.

40. R. R. Yager and J. Kacprzyk, editors. The Ordered Weighted Averaging Operators: Theory and Applications. Kluwer Academic Publishers, Norwell, MA, USA, 1997. 